\title{
Circuit
}

Musiques contemporaines

\section{Le Quatuor selon Schafer... et selon d'autres}

\section{Jean Portugais}

Volume 11, numéro 2, 2000

Le quatuor à cordes selon Schafer

URI : https://id.erudit.org/iderudit/004687ar

DOI : https://doi.org/10.7202/004687ar

Aller au sommaire du numéro

Éditeur(s)

Les Presses de l'Université de Montréal

ISSN

1183-1693 (imprimé)

1488-9692 (numérique)

Découvrir la revue

Citer cet article

Portugais, J. (2000). Le Quatuor selon Schafer... et selon d'autres. Circuit, 11(2),

7-10. https://doi.org/10.7202/004687ar d'utilisation que vous pouvez consulter en ligne.

https://apropos.erudit.org/fr/usagers/politique-dutilisation/ 


\title{
Le Quatuor selon Schafer... et selon d'autres
}

\author{
Jean Portugais, rédacteur invité
}

En décembre 1999, Olga Ranzenhofer et son Quatuor Molinari ont organisé un événement de trois jours autour du compositeur canadien R. Murray Schafer et de ses quatuors à cordes. Plutôt que de se limiter au rôle d'interprètes de la musique d'aujourd'hui et d'un hier encore récent, les Molinari ont décidé de jouer un rôle d'animateur culturel en proposant une conférence, des ateliers, une table ronde et un concert coordonnés.

Cet événement, qui s'est conclu par la première intégrale à être réalisée en un concert marathon des sept quatuors à cordes de Schafer, a bénéficié à Montréal d'une forte participation du public et d'une couverture médiatique assez exceptionnelle pour de la musique de chambre contemporaine. C'est que le Quatuor Molinari se préparait, depuis sa fondation trois ans plus tôt, à jover ces $150 \mathrm{mi}-$ nutes de musique au cours d'une seule soirée. Pour l'occasion, le Quatuor Molinari a créé la version scénique du Septième Quatuor de Schafer. L'ensemble, exclusivement dédié au répertoire du Xxe siècle, jouait donc régulièrement Schafer à ses concerts parmi de multiples autres œeuvres du répertoire, de Webern et Bartók à Scelsi et Schnittke, en passant par Chostakovitch, Evangelista, Glass, Gougeon, Korngold, Kurtág, Ligeti, Louie, Martinu, Sokolovic et plusieurs autres. Cette ouverture et cette diversité esthétique chez un ensemble traditionnel comme le quatuor à cordes n'est pas chose courante. Et le fait que des musiciens professionnels choisissent de se centrer pendant des mois sur un seul compositeur contemporain n'est pas non plus une chose fréquente dans les sociétés de concert actuelles. En outre, lors de ces journées, les musiciens ont présenté en public une analyse des quatuors, et ce, en présence du compositeur. L'auditoire était invité à poser des questions sur la composition et sur l'interprétation de ces œuvres.

Pourtant, la dimension de l'œuvre pour quatuor de Schafer parle d'elle-même ; pour s'en convaincre, il suffit de rappeler ici que de nombreux autres ensembles canadiens jouent des quatuors de Schafer depuis près de trente ans. Le Quatuor Orford a donné fréquemment en concert et un peu partout dans le monde les cinq premiers quatuors (qu'il a d'ailleurs enregistrés en 1990, peu avant la cessation 
des activités du groupe), tandis que le Quatuor Morency a réalisé en 1989, à la SMCQ, une présentation des Quatuors nos 1 à 3. Ailleurs au Canada et aux États-Unis, le Quatuor Penderecki, le Quatuor Purcell et le Quatuor St. Lawrence ont aussi régulièrement joué différents quatuors de Schafer. Cependant, jamais une intégrale comme celle du Quatuor Molinari n'avait été réalisée en un seul concert.

C'est à cette occasion que Olga Ranzenhofer, membre fondatrice du Quatuor Molinari et directrice des activités de l'ensemble, m'a demandé de préparer un volet public comprenant une conférence de Murray Schafer et une table ronde réunissant des compositeurs et des musicologues québécois autour de la question de l'actualité du quatuor à cordes.

Le présent numéro de Circuit, musiques contemporaines est principalement consacré aux résultats écrits découlant de ces activités que nous avions nommées "Le Quatuor selon Schafer». Le dossier que j'ai réuni dans ce numéro contient les parties suivantes: la conférence de Schafer du 9 décembre 1999; un parcours dans l'œuvre pour quatuor à cordes de Schafer, intitulé «îles de la Nuit», que Olga Ranzenhofer et moi-même avons corédigé; et les développements de la table ronde centrée sur la question: "Le quatuor est-il une forme tabou, un fossile ou appartient-il encore à une tradition vivante? ». Les autres éléments du présent numéro ont été préparés par le rédacteur en chef Michel Duchesneau qui, en plus de veiller sans relâche aux conditions de réalisation de ce numéro, a eu l'heureuse initiative de proposer des chroniques complémentaires au dossier comme la bibliographie sélective sur Murray Schafer, confiée à Jonathan Goldman, ou encore sa propre recension du livre de Bernard Fournier, consacrée à l'esthétique du quatuor à cordes classique.

Que soient chaleureusement remerciés ici Murray Schafer et les membres du Quatuor Molinari pour leur soutien dans la préparation et la réalisation de cette entreprise à la fois vécue et écrite. Je remercie vivement Michel Duchesneau pour ses généreux efforts, son ouverture d'esprit et ses initiatives concernant ce numéro. Avec aisance, il a réussi à mener à terme une préparation de numéro thématique initiée par son prédécesseur Jean Boivin. Je remercie d'ailleurs Jean Boivin et JeanJacques Nattiez (tous deux ex-rédacteurs en chef de Circuit) qui ont montré un intérêt constant pour le présent dossier et qui m'ont encouragé à le soumettre à Circuit, musiques contemporaines. Un merci tout spécial à la secrétaire de rédaction, Johanne Rivest, pour son très fin travail durant la préparation des épreuves. Pour leur participation à ce numéro thématique, je remercie également les auteurs des textes de la table ronde, Jean Boivin, José Evangelista, Jean-Jaques Nattiez, Serge Provost et John Rea. Je souhaite aussi remercier le comité de rédaction de la revue pour son travail sur les textes. Enfin, je suis reconnaissant à la directrice administrative, Suzanne Samson, de sa collaboration aux démarches concernant l'édition et l'illustration du numéro. 
La pérennité du quatuor à cordes est parfois mise en doute. Je souhaite que le présent dossier puisse apporter la preuve de sa vitalité comme lieu de création contemporaine, ne serait-ce que par la passion et la controverse que cette question suscite dans les différents textes qui sont ici réunis. 


\section{FOURTH STRING QUARTET}

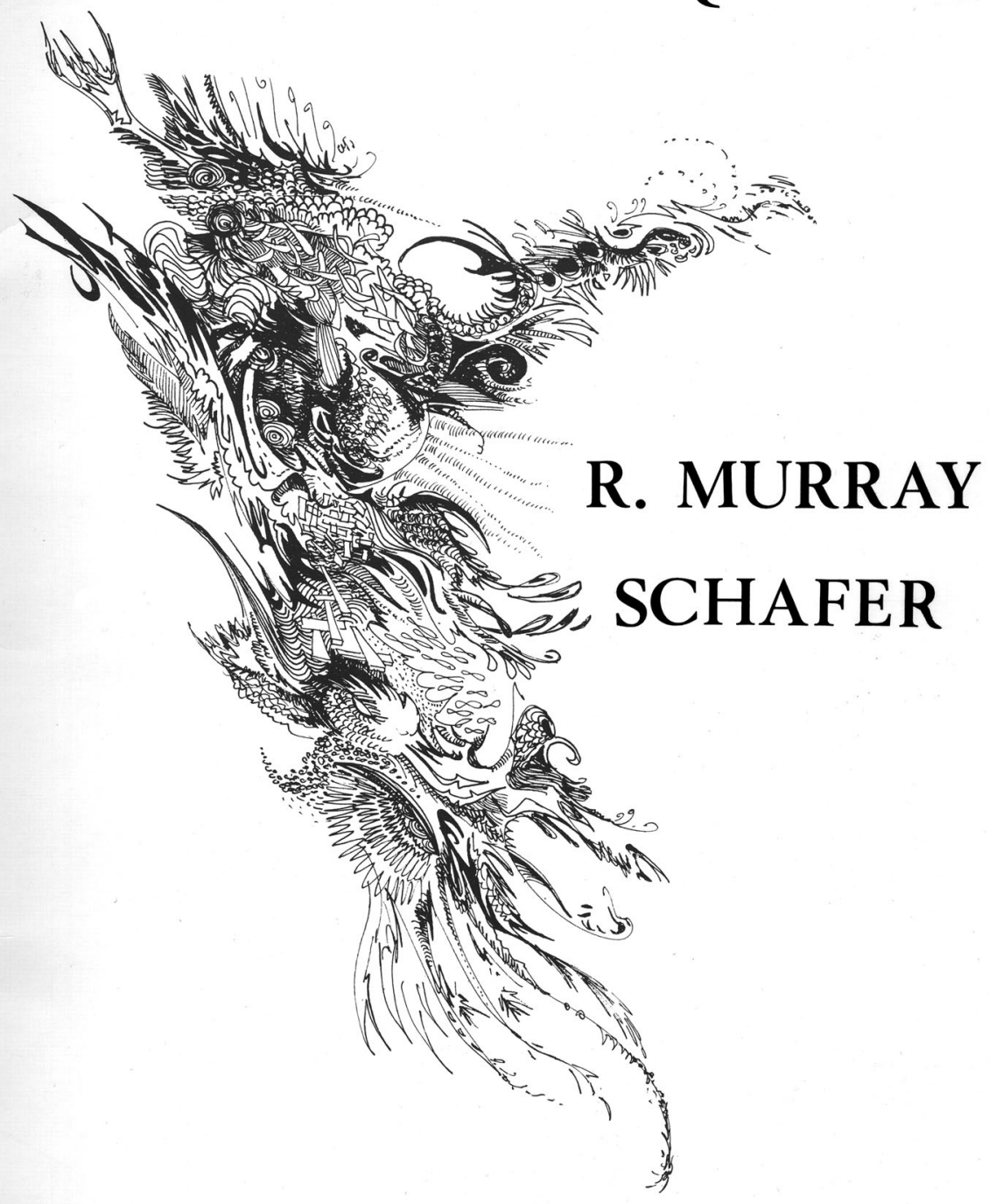

